J Urol. 2014 January ; 191(1): 169-174. doi:10.1016/j.juro.2013.07.086.

\title{
Impact of the 2012 American Urological Association Vasectomy Guidelines on Post-Vasectomy Outcomes
}

\author{
Robert M. Coward, Niraj G. Badhiwala, Jason R. Kovac, Ryan P. Smith, Dolores J. Lamb, \\ and Larry I. Lipshultz ${ }^{*} \dagger$ \\ Scott Department of Urology, Baylor College of Medicine, Houston, Texas
}

\begin{abstract}
Purpose-The 2012 American Urological Association (AUA) vasectomy guidelines recommend the finding of rare nonmotile sperm, representing 100,000 or fewer nonmotile sperm per $\mathrm{ml}$, as a metric of post-vasectomy success. At our institution success was previously defined as 2 sequential azoospermic centrifuged semen pellets. The criteria change of including rare nonmotile sperm as a success end point may simplify post-vasectomy followup and decrease the number of post-vasectomy semen analyses required to assure occlusive success.
\end{abstract}

Materials and Methods-In the context of the new 2012 guidelines we retrospectively reviewed and analyzed the records of 972 of the 1,740 vasectomies (55.9\%) performed between January 2000 and June 2012 after which at least 1 post-vasectomy semen analysis was done.

Results-A total of 1,919 post-vasectomy semen analyses were obtained from 972 patients with a mean \pm SE age of $39.7 \pm 0.2$ years. Occlusive success was evident in 337 azoospermic men $(36.4 \%)$, while $514(52.9 \%)$ underwent 2 or more post-vasectomy semen analyses and 458 (47.1\%) returned for a single post-vasectomy semen analysis but were lost to followup. Of these noncompliant patients $76.0 \%$ were azoospermic, $19.7 \%$ had rare nonmotile sperm, $1.5 \%$ had greater than 100,000 nonmotile sperm per $\mathrm{ml}$ and $2.8 \%$ had motile sperm. Three patients underwent repeat vasectomy for persistent rare nonmotile sperm. If the criteria defined by the 2012 guidelines had been used to monitor these men, the occlusive success rate would have improved to $97.6 \%$ (949 patients) ( $\mathrm{p}<0.05$ ). Repeat vasectomies as well as 896 subsequent postvasectomy semen analyses would have been avoided.

Conclusions-The AUA vasectomy guidelines provide clear, evidence-based criteria for vasectomy success. The guidelines simplify followup protocols, improve patient compliance and help avoid unnecessary post-vasectomy semen analyses and repeat vasectomies.

\section{Keywords}

testis; sterilization; reproductive; semen analysis; reoperation; guideline

\footnotetext{
“Correspondence: 6624 Fannin St., Suite 1700, Houston, Texas 77030 (telephone: 713-798-6163; FAX: 713-798-6007; larryl@bcm.edu)..

${ }^{\dagger}$ Financial interest and/or other relationship with Eli Lilly, Endo, Auxilium, Pfizer, Allergan and American Medical Systems. Study received approval from the Baylor College of Medicine institutional review board for the protection of human subjects.
} 
$\mathrm{V}_{\text {ASECTOMY }}$ is the most common urological procedure in the United States with an estimated 500,000 or more performed annually. ${ }^{1,2}$ Although it is effective, safe and more cost efficient with faster convalescence than female sterilization, vasectomy is the second most common form of permanent sterilization. ${ }^{3}$ In 2012 a panel comprising experts from the AUA defined evidence-based vasectomy guidelines. ${ }^{4}$ These guidelines are likely to positively impact practice patterns for clinicians who perform vasectomy.

The failure rate after vasectomy with a subsequent risk of pregnancy after documented occlusive success is approximately $1 / 2,000$ cases. ${ }^{5}$ Vasectomy has the lowest failure rate of all forms of contraception. ${ }^{6}$ Groups at many clinics adhere to strict practice protocols, such that azoospermic PVSA based on assessment of a centrifuged pellet ensures the likelihood of sterility. Due to the variable clearance time of residual sperm from the male genital tract, PVSA timing, the number of PVSAs required to achieve azoospermia and the number of ejaculations after vasectomy do not correlate well with the achievement of azoospermia after vasectomy. ${ }^{7,8}$ For these reasons, at our andrology laboratory as well as at others confirmation of sterility after vasectomy was defined as azoospermia on 2 sequential PVSAs with concurrent centrifuged pellet analyses. ${ }^{9-13}$

In contrast to these more conservative protocols of post-vasectomy followup, the AUA vasectomy guidelines define vasectomy success as azoospermia or RNMS in a single uncentrifuged PVSA, defined as 100,000 or greater nonmotile sperm per $\mathrm{ml} .{ }^{4}$ Compared with our laboratory defined PVSA criteria of azoospermia on pellet analysis, including RNMS in the definition of occlusive success has the potential to decrease the number of PVSAs, improve the documented success rate, increase compliance with followup and reduce the number of repeat vasectomies.

We determined how the less stringent 2012 AUA vasectomy guidelines would have altered our clinical practice. Accordingly, we analyzed our PVSA data to determine whether our clinical practice outcomes would have changed in light of the new criteria.

\section{MATERIALS AND METHODS}

This study was approved by the institutional review board for the protection of human subjects at Baylor College of Medicine. We retrospectively reviewed the records of all men who underwent vasectomy and returned for at least 1 PVSA from January 2000 to June 2012. All PVSA data were analyzed. The charts of all men who were not cleared as sterile by our institutional followup protocol were reviewed to determine whether repeat vasectomy was performed or unintended pregnancy occurred.

All vasectomies were performed by a single surgeon after obtaining informed consent. The vasectomy technique was conventional vasectomy using bilateral upper scrotal skin incisions. Intravenous sedation and local anesthesia were used. Vasal occlusion was achieved by placing titanium clips and removing a vasal segment.

Informed consent included the institutional policy recommendation that the patient provide 2 sequential PVSAs at least 1 week apart beginning 3 months after vasectomy. A masturbatory 
sample was collected in the office or brought in and examined within 1 hour of collection. No appointments were scheduled and the patient was responsible for providing the samples.

Each PVSA was initially evaluated as a complete semen analysis performed manually by a certified andrology laboratory technician. If the sample was azoospermic, it was centrifuged at $200 \times$ gravity for 10 minutes for semen pellet analysis. Documentation of 2 sequential azoospermic PVSAs using a protocol requiring analysis of the centrifuged semen pellet was used to define the occlusive success rate and patient compliance with the post-vasectomy followup protocol of our practice. For patients who underwent repeat vasectomy the sperm concentration and motility was recorded from each PVSA.

To determine how the revised practice criteria based on the 2012 AUA vasectomy guidelines would have changed post-vasectomy outcomes, each patient was reevaluated to assess compliance and the vasectomy occlusive success rate as well as the need for repeat vasectomy according to the new criteria. Since each PVSA included data on routine manual semen analysis as well as centrifuged pellet analysis, accurate comparison was possible between our standard andrology laboratory protocol using centrifuged pellet analysis and the AUA vasectomy guidelines using uncentrifuged semen analysis.

Time to occlusive success was determined by the days between the first PVSA and documented occlusive success on a subsequent PVSA. Because all men were required to wait at least 3 months before the first PVSA, this time was not calculated into the difference between the time to clearance for the institutional protocol vs the guideline protocol. The number of repeat PVSAs that would no longer be considered necessary based on the revised criteria of the guidelines, the estimated cost of any unneeded PVSAs in men who did not achieve azoospermia but had RNMS and the number of repeat vasectomies were determined to ascertain the cumulative benefit of using the new guidelines.

We used 2-tailed z-test calculations to determine statistical significance between our established andrology laboratory definition and the AUA vasectomy guidelines. Data were analyzed using Excel@ and SPSS ${ }^{\circledR}$ with differences considered statistically significant at $\mathrm{p}$ $<0.05$.

\section{RESULTS}

A total of 1,740 vasectomies were performed between January 2000 and June 2012. The study cohort comprised 972 men (55.9\%) who returned to our laboratory for at least 1 PVSA. A total of 1,919 PVSAs were obtained in these men, who had a mean \pm SE age of $39.7 \pm 0.2$ years. Initial PVSA performed 3 months after vasectomy in the 972 tested men revealed that $643(66.2 \%)$ were azoospermic, 278 (28.6\%) presented with RNMS, 34 (3.5\%) had greater than 100,000 nonmotile sperm per $\mathrm{ml}$ and 17 (1.8\%) had motile sperm present in the ejaculate (part $A$ of figure). Of the 972 men who returned for the initial PVSA 458 (47.1\%) never returned for the second PVSA, 333 (34.3\%) returned for the second PVSA and $181(18.6 \%)$ required 3 or more PVSAs because sperm continued to be present in the ejaculate. Of the 458 men with a single PVSA who did not return for followup 348 (76.0\%) were azoospermic, $90(19.7 \%)$ had RNMS, 7 (1.5\%) had greater than 100,000 nonmotile 
sperm per $\mathrm{ml}$ and $13(2.8 \%)$ had motile sperm in the ejaculate. Due to the retrospective nature of the study we did not contact men with more than 100,000 nonmotile sperm per ml or men with motile sperm who were lost to followup. In all patients mean time between the first and second PVSAs was 56.2 days (median 19) with a mean of 76.3 days (median 28) between the second and third PVSAs.

The documented occlusive success rate in our study population, as defined by our andrology laboratory protocol requiring 2 sequential PVSAs confirming azoospermia on a centrifuged pellet, was $34.7 \%$ (337 men) in those with at least 1 PVSA. These data also represent patient compliance with our recommended followup. Despite this low documented occlusive success rate and patient compliance no unintended pregnancy was noted in the cohort or reported by patients lost to followup. Thus, the known contraceptive success rate was $100 \%$.

Three patients $(0.002 \%)$ underwent repeat vasectomy for persistent RNMS in the ejaculate long after the vasectomy. In all 3 patients the last PVSA revealed azoospermia on initial semen analysis and only identified RNMS on centrifuged pellet analysis before repeat vasectomy. On subsequent PVSA after repeat vasectomy 2 of the 3 patients continued to show RNMS. Another PVSA was not done thereafter in the third patient.

Based on the 2012 AUA vasectomy guidelines, which recommend only a single uncentrifuged PVSA finding of azoospermia or RNMS, repeat data analysis revealed that the occlusive success rate and, thus, compliance improved to 97.6\% (949 patients) ( $\mathrm{p}<0.001$, see table). A total of 921 patients (94.7\%) with azoospermia or RNMS on the first PVSA met the criteria for occlusive success, while another 28 achieved azoospermia or RNMS on the second PVSA (part $B$ of figure). The remaining patients with greater than 100,000 nonmotile sperm per $\mathrm{ml}$ or any motile sperm were lost to followup. On the first PVSA 897 patients $(92.3 \%)$ were azoospermic on initial uncentrifuged semen analysis, as would be recommended by the guidelines. However, in 254 of those men (28.3\%) RNMS was identified on centrifuged pellet analysis only and they were not cleared based on the institutional protocol. Also, all 3 repeat vasectomies were done due to continued RNMS but they would have been unnecessary based on the criteria for success defined by the guidelines. While it might be predicted that no unintended pregnancies would have occurred based on these criteria, it is likely that many of these men continued to use some form of contraception.

A total of 1,919 combined PVSAs were performed. When data were reanalyzed according to the 2012 AUA vasectomy guidelines, 896 PVSAs would not have been necessary (see table). PVSA with centrifuged pellet analysis currently costs $\$ 150$ at our institution. Therefore, if the AUA vasectomy guidelines had been used throughout the study period, approximately $\$ 134,400$ in total health care expenditure would have been saved.

Based on the criteria used for the 2012 AUA vasectomy guidelines the men in this study could have returned to unprotected intercourse much earlier. A total of 337 patients who underwent more than 1 PVSA for RNMS would otherwise have been cleared for unprotected intercourse right away under the 2012 guidelines. However, after the first PVSA they returned for an additional PVSA at a mean \pm SD of $227.7 \pm 487.4$ days (mean \pm SE 227.7 
\pm 26.4 , median 21), resulting in lost work hours. On reanalysis according to the guidelines occlusive success was achieved in 949 men at a mean \pm SD of $3.0 \pm 24.4$ days (mean $\pm \mathrm{SE}$ of $3.0 \pm 0.8$, median 0 ) after the first PVSA. Accordingly, a significant time savings would have been realized in men using alternative forms of contraception for a much longer period after vasectomy.

\section{DISCUSSION}

Vasectomy provides a reliable method of contraception because obstruction of the vas deferens prevents sperm from passing completely through the genital tract into the ejaculate. Since the procedure goals are to achieve azoospermia and contraception, traditional approaches used to define vasectomy success focus on azoospermia as the final end point.

Evidence-based data led the AUA vasectomy guideline committee to include RNMS in the ejaculate in the definition of vasectomy occlusive success. 1) The percent of men who ultimately achieve complete azoospermia after vasectomy varies. ${ }^{7,8}$ Although the failure rate has remained constantly low at 1/2,000 men, ${ }^{5}$ PVSA studies reveal that up to $33 \%$ have RNMS remaining in the ejaculate 12 weeks after vasectomy. ${ }^{14}$ 2) Performing 2 consecutive azoospermic PVSAs is not critical because it is possible for sperm to return to the ejaculate when there is late recanalization, even after 2 consecutive azoospermic PVSAs. ${ }^{5,14}$ Lemack and Goldstein analyzed preoperative semen samples of 186 sterile, vasectomized men before vasectomy reversal with an average of $10.7 \pm 5.6$ years since vasectomy. ${ }^{15}$ Of the men 16 (8.6\%) had RNMS, while 2 (1.1\%) had motile sperm in the ejaculate. 3) Perhaps the most important point is that men with RNMS show a contraceptive failure rate similar to that of azoospermic men after vasectomy. ${ }^{11,16-18}$ Even when motile sperm are found in the first PVSA, more than $50 \%$ of patients ultimately achieve delayed success. ${ }^{19}$

The purpose of this study was to compare our traditional approach to post-vasectomy determination of occlusive success with that of the 2012 AUA vasectomy guidelines. ${ }^{4}$ With the recent introduction of RNMS into the definition of occlusive success we hypothesized that the guidelines would decrease the number of PVSAs, thus decreasing costs, improving the success rate and compliance with followup, and ultimately reducing the number of repeat vasectomies.

Of the 1,740 vasectomies performed only 972 men (55.9\%) returned for at least 1 PVSA. Several previous studies showed a significant noncompliance rate of men returning for the recommended PVSA, comparable with our cohort. ${ }^{9,20,21}$ For example, in a series of 1,892 consecutive vasectomies $34 \%$ of men did not return and 33\% returned for only 1 of 2 recommended PVSAs. ${ }^{9}$ Another series of 1,029 vasectomies showed that only $54.4 \%$ of men returned for 2 recommended PVSAs. ${ }^{20}$ Compliance is higher if a followup appointment is made by the office. ${ }^{22}$ The primary issue identified in studies of post-vasectomy followup compliance is failure to return for additional PVSAs when sperm are found in the initial sample. Our data indicate that subsequent PVSAs are rarely required under the new guidelines. 
Although 3 patients in our cohort underwent repeat vasectomy for persistent RNMS, the putative vasectomy failure was established by centrifuged pellet analysis. Based on the current guidelines all 3 patients would have avoided repeat vasectomy. Even after repeat vasectomy 2 men continued to have RNMS in the ejaculate, while the other did return for PVSA after repeat vasectomy. These results suggest that RNMS after vasectomy arise from the distal or abdominal side of the vas deferens, the ampulla of the vas deferens or the seminal vesicles. To our knowledge no evidence shows that RNMS indicate a persistently patent vas deferens or recanalization.

Using class C evidence the 2012 AUA vasectomy guidelines recommend that the vas should be occluded by mucosal cautery with fascial interposition and without clips, mucosal cautery without fascial interposition and without clips, open ended vasectomy with fascial interposition and mucosal cautery on the abdominal end or the nondivisional method of extended electrocautery. ${ }^{4}$ In our study $100 \%$ contraceptive success was achieved by removing a vasal segment and applying clips without fascial interposition. The guidelines include a caveat that vasal occlusion with clips with or without fascial interposition and with or without excision of a vasal segment may be performed by experienced surgeons. At our center conventional vasectomy has been performed with removal of a vasal segment, application of clips to each end and without fascial interposition for decades with excellent results.

Our study revealed that 896 PVSAs would have been avoided under the guidelines with an estimated cost savings of $\$ 134,400$. Thus, by defining vasectomy success as 2 sequential azoospermic PVSAs with concurrent pellet analysis $46.5 \%$ more PVSAs were performed in our cohort than if the criteria of the guidelines had been used. In contrast to British Andrology Society guidelines, which also recommend 2 consecutive azoospermic PVSAs, a prospective study of 832 men in the United Kingdom reported a cost savings of $40 \%$ by only requiring 1 PVSA without compromising the outcome. ${ }^{23}$ We similarly present a potential cost savings of $46.7 \%$ by following the AUA vasectomy guidelines and including RNMS in the definition of occlusive success, also without a change in the contraceptive success rate.

\section{CONCLUSIONS}

The AUA vasectomy guidelines provide clear, evidence-based criteria to determine vasectomy success, which include azoospermia or RNMS in a single uncentrifuged PVSA. The guidelines simplify followup protocols, improve patient compliance and save cost and time by avoiding unnecessary subsequent PVSAs and repeat vasectomy.

\section{Abbreviations and Acronyms \\ PVSA post-vasectomy semen analysis \\ RNMS rare nonmotile sperm}




\section{REFERENCES}

1. Barone MA, Hutchinson PL, Johnson CH, et al. Vasectomy in the United States, 2002. J Urol. 2006; 176:232. [PubMed: 16753407]

2. Eisenberg ML, Lipshultz LI. Estimating the number of vasectomies performed annually in the United States: data from the National Survey of Family Growth. J Urol. 2010; 184:2068. [PubMed: 20850832]

3. Martinez GM, Chandra A, Abma JC, et al. Fertility, contraception, and fatherhood: data on men and women from cycle 6 (2002) of the 2002 National Survey of Family Growth. Vital Health Stat. 2006; 23:1.

4. Sharlip ID, Belker AM, Honig S, et al. Vasectomy: AUA guideline. J Urol. 2012; 188:2482. [PubMed: 23098786]

5. Philp T, Guillebaud J, Budd D. Late failure of vasectomy after two documented analyses showing azoospermic semen. Br Med J (Clin Res Ed). 1984; 289:77.

6. Trussell J. Contraceptive failure in the United States. Contraception. 2011; 83:397. [PubMed: 21477680]

7. Barone MA, Irsula B, Chen-Mok M, et al. Effectiveness of vasectomy using cautery. BMC Urol. 2004; 4:10. [PubMed: 15260885]

8. Barone MA, Nazerali H, Cortes M, et al. A prospective study of time and number of ejaculations to azoospermia after vasectomy by ligation and excision. J Urol. 2003; 170:892. [PubMed: 12913724]

9. Maatman TJ, Aldrin L, Carothers GG. Patient noncompliance after vasectomy. Fertil Steril. 1997; 68:552. [PubMed: 9314934]

10. Bradshaw HD, Rosario DJ, James MJ, et al. Review of current practice to establish success after vasectomy. Br J Surg. 2001; 88:290. [PubMed: 11167883]

11. Davies AH, Sharp RJ, Cranston D, et al. The long-term outcome following "special clearance" after vasectomy. Br J Urol. 1990; 66:211. [PubMed: 2390708]

12. Dhar NB, Bhatt A, Jones JS. Determining the success of vasectomy. BJU Int. 2006; 97:773. [PubMed: 16536771]

13. Hancock P, McLaughlin E. British Andrology Society guidelines for the assessment of post vasectomy semen samples (2002). British Andrology Society. J Clin Pathol. 2002; 55:812. [PubMed: 12401817]

14. De Knijff DW, Vrijhof HJ, Arends J, et al. Persistence or reappearance of nonmotile sperm after vasectomy: does it have clinical consequences? Fertil Steril. 1997; 67:332. [PubMed: 9022612]

15. Lemack GE, Goldstein M. Presence of sperm in the pre-vasectomy reversal semen analysis: incidence and implications. J Urol. 1996; 155:167. [PubMed: 7490823]

16. Korthorst RA, Consten D, van Roijen JH. Clearance after vasectomy with a single semen sample containing < than 100000 immotile sperm/mL: analysis of 1073 patients. BJU Int. 2010; 105:1572. [PubMed: 20002679]

17. Edwards IS. Earlier testing after vasectomy, based on the absence of motile sperm. Fertil Steril. 1993; 59:431. [PubMed: 8425641]

18. Jouannet P, David G. Evolution of the properties of semen immediately following vasectomy. Fertil Steril. 1978; 29:435. [PubMed: 648646]

19. Labrecque M, St-Hilaire K, Turcot L. Delayed vasectomy success in men with a first postvasectomy semen analysis showing motile sperm. Fertil Steril. 2005; 83:1435. [PubMed: 15866581]

20. Belker AM, Sexter MS, Sweitzer SJ, et al. The high rate of noncompliance for post-vasectomy semen examination: medical and legal considerations. J Urol. 1990; 144:284. [PubMed: 2374193]

21. Sheynkin Y, Mishail A, Vemulapalli P, et al. Sociodemographic predictors of postvasectomy noncompliance. Contraception. 2009; 80:566. [PubMed: 19913151]

22. Dhar NB, Jones JS, Bhatt A, et al. A prospective evaluation of the impact of scheduled follow-up appointments with compliance rates after vasectomy. BJU Int. 2007; 99:1094. [PubMed: 17233802] 
23. Senanayake E, Pacey AA, Maddireddy V, et al. A novel cost-effective approach to post-vasectomy semen analysis. BJU Int. 2011; 107:1447. [PubMed: 21388491] 
A
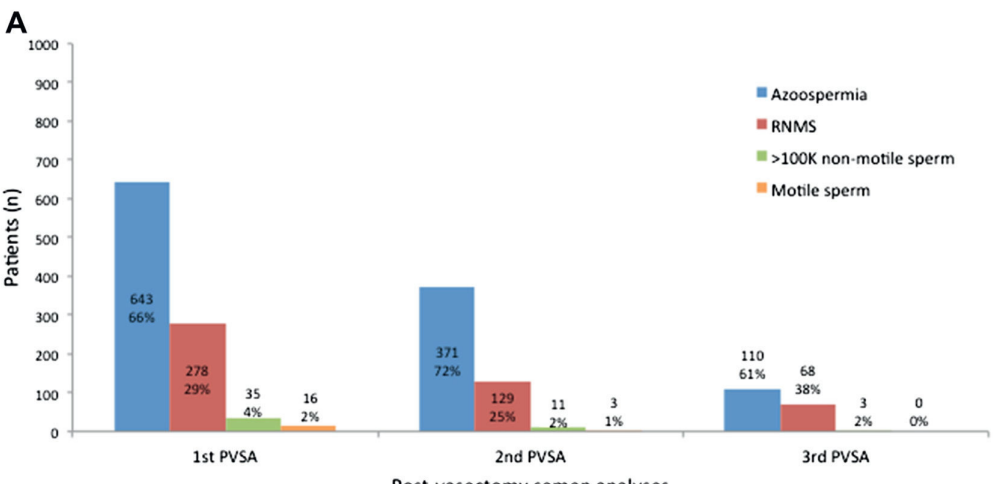

B

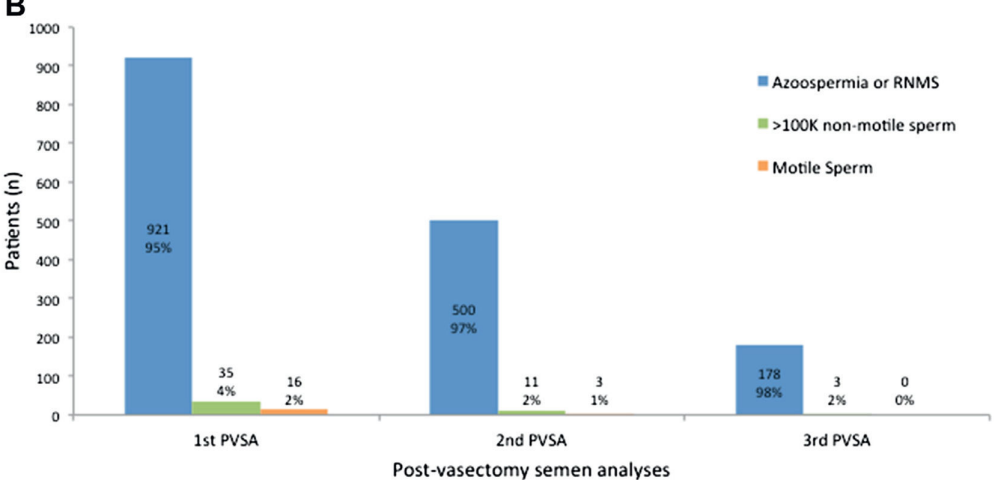

figure.

Findings at PVSA 1 to 3 in all patients. $A$, azoospermia, RNMS, greater than 100,000 nonmotile sperm and any motile sperm. $B$, azoospermia plus RNMS, indicating occlusive success according to AUA vasectomy guidelines, vs greater than 100,000 nonmotile sperm or any motile sperm. 
Institutional laboratory post-vasectomy followup protocol vs AUA vasectomy guidelines in 972 patients each

\begin{tabular}{|c|c|c|c|}
\hline & Institutional* & AUA* & Difference \\
\hline No. PVSAs ${ }^{\dagger}$ & 1,919 & 1,023 & 896 \\
\hline$\%$ Occlusive success (No. pts) ${ }^{\dagger}:$ & $34.7(337)$ & $97.6(949)$ & 62.9 \\
\hline No. repeat vasectomies ${ }^{\dagger}$ & 3 & 0 & 3 \\
\hline Total PVSA cost $(\$)^{\dagger}$ & 287,850 & 153,450 & 134,400 \\
\hline Mean/median days to occlusive success after PVSA $1^{\dagger}$ & $227.7 / 21$ & $3.0 / 0$ & 21 \\
\hline
\end{tabular}

* No unintended pregnancy with contraception achieved in all patients.

$t_{\mathrm{p}}<0.001$. 